Research Article

\title{
A New Efficient Method for Solving Two-Dimensional Nonlinear System of Burger's Differential Equations
}

\author{
Shams A. Ahmed $D^{1},{ }^{1}$ Mohamed Elbadri $\left(D,{ }^{1}\right.$ and Mohamed Z. Mohamed ${ }^{2}$ \\ ${ }^{1}$ Department of Mathematic, Faculty of Sciences and Arts, Jouf University, Tubarjal, Saudi Arabia \\ ${ }^{2}$ Department of Mathematic, Deanship of Preparatory Programs, University of Hail, Hail, Saudi Arabia
}

Correspondence should be addressed to Shams A. Ahmed; shamsalden20@hotmail.com

Received 29 October 2019; Revised 29 December 2019; Accepted 7 January 2020; Published 11 February 2020

Academic Editor: Kunquan Lan

Copyright (c) 2020 Shams A. Ahmed et al. This is an open access article distributed under the Creative Commons Attribution License, which permits unrestricted use, distribution, and reproduction in any medium, provided the original work is properly cited.

\begin{abstract}
In this work, the Sumudu decomposition method (SDM) is utilized to obtain the approximate solution of two-dimensional nonlinear system of Burger's differential equations. This method is considered to be an effective tool in solving many problems. Our results have shown that the SDM offers a much better approximation for solving several numbers of systems of twodimensional nonlinear Burger's differential equations. To clarify the facility and accuracy of the strategy, two examples are provided.
\end{abstract}

\section{Introduction}

Burger's equation is one of the foremost necessary partial differential equations in fluid mechanics. This equation demonstrates the coupling between diffusion and convection processes. Burger's equation describes the structure of shock waves, traffic flow, and acoustic transmission. Additionally, like this, it also appears in varied areas of applied mathematics and physics, such as modelling of gas dynamics [1-5]. Recently, many numerical and analytical methods have been used to study the two-dimensional Burger's equation such as the differential transformation method [6], homotopy perturbation method [7], homotopy analysis method [8], variational iteration method [9], Adomian decomposition method [10-12], cubic B-spline differential quadrature method [13], finite difference method [14], finite element [15], and local discontinuous Galerkin finite element method [16] and also mathematicians have used transform methods coupled with analytical methods [17-30] to solve PDEs. The Sumudu decomposition method (SDM) is one of these methods, and it has been successfully used to solve intricate problems in engineering mathematics and applied science [31-35]. The SDM was first introduced by Kumar [36], to solve nonlinear partial differential equations that show in all aspects of applied science and engineering. This method is an elegant combination of the Sumudu transform method and the Adomian decomposition method. The SDM method generates the solution in a series form whose components are determined by a recursive relationship.

In the current study, we consider the system of twodimensional nonlinear Burger's equations [9]:

$$
\begin{gathered}
\theta_{t}+\theta \theta_{\rho}+\alpha \theta_{\sigma}=\frac{1}{\mathbf{R}}\left(\theta_{\rho \rho}+\theta_{\sigma \sigma}\right), \\
\alpha_{t}+\theta \alpha_{\rho}+\alpha \alpha_{\sigma}=\frac{1}{\mathbf{R}}\left(\alpha_{\rho \rho}+\alpha_{\sigma \sigma}\right),
\end{gathered}
$$

with the initial conditions:

$$
\begin{aligned}
& \theta(\rho, \sigma, 0)=w(\rho, \sigma), \rho, \sigma \in E, \\
& \alpha(\rho, \sigma, 0)=h(\rho, \sigma), \rho, \sigma \in E,
\end{aligned}
$$


and the boundary conditions:

$$
\begin{aligned}
& \theta(\rho, \sigma, t)=w_{1}(\rho, \sigma, t), \rho, \sigma \in \partial E, \\
& \alpha(\rho, \sigma, t)=h_{1}(\rho, \sigma, t), \rho, \sigma \in \partial E,
\end{aligned}
$$

where $E=\{(\rho, \sigma) \mid a \leq \rho \leq b, a \leq \sigma \leq b\}$ and $\partial E$ is its boundary, $\theta(\rho, \sigma, t)$ and $\alpha(\rho, \sigma, t)$ are the velocity components to be determined, $w, h, w_{1}$, and $h_{1}$ are the known functions, and $R$ is the Reynolds number.

The major objective of this work is to get analytical and numerical solutions of the system of two-dimensional nonlinear Burger's equations (1) by using SDM. This work is organized as follows: the analysis of the method is given in Section 2. The application of SDM to two examples is given in Section 3. Concluding remarks are given in the last section.

\section{Analysis of the Method}

Now, to obtain the approximate solution of equation (1), apply the Sumudu transformation to equation (1) and using the given condition (2) gives

$$
\begin{aligned}
& S[\theta(\rho, \sigma, u)]=w(\rho, \sigma)-u S\left[\theta \theta_{\rho}+\alpha \theta_{\sigma}\right]+u S\left[\frac{1}{R}\left(\nabla^{2} \theta\right)\right], \\
& S[\alpha(\rho, \sigma, u)]=h(\rho, \sigma)-u S\left[\theta \alpha_{\rho}+\alpha \alpha_{\sigma}\right]+u S\left[\frac{1}{R}\left(\nabla^{2} \alpha\right)\right],
\end{aligned}
$$

where $\nabla^{2}=\left(\partial^{2} / \partial \rho^{2}\right)+\left(\partial^{2} / \partial \sigma^{2}\right)$. Apply the inverse operator $S^{-1}$ to both sides of the equation (4), and it gives

$$
\begin{aligned}
& \theta(\rho, \sigma, t)=w(\rho, \sigma)-S^{-1}\left[u S\left[\theta \theta_{\rho}+\alpha \theta_{\sigma}\right]\right]+S^{-1}\left[u S\left[\frac{1}{R}\left(\nabla^{2} \theta\right)\right]\right], \\
& \alpha(\rho, \sigma, t)=h(\rho, \sigma)-S^{-1}\left[u S\left[\theta \alpha_{\rho}+\alpha \alpha_{\sigma}\right]\right]+S^{-1}\left[u S\left[\frac{1}{R}\left(\nabla^{2} \alpha\right)\right]\right] .
\end{aligned}
$$

The Adomian decomposition method suggests that the linear terms $\theta(\rho, \sigma, t)$ and $\alpha(\rho, \sigma, t)$ and the nonlinear terms $\theta \theta_{\rho}, \alpha \theta_{\sigma}, \theta \alpha_{\rho}$, and $\alpha \alpha_{\sigma}$ are decomposed by an infinite series of components:

$$
\begin{aligned}
\theta(\rho, \sigma, t) & =\sum_{n=0}^{\infty} \theta_{n}(\rho, \sigma, t), \\
\theta \theta_{\rho} & =\sum_{n=0}^{\infty} A_{n}, \\
\alpha \theta_{\sigma} & =\sum_{n=0}^{\infty} B_{n}, \\
\alpha(\rho, \sigma, t) & =\sum_{n=0}^{\infty} \alpha_{n}(\rho, \sigma, t), \\
\theta \alpha_{\rho} & =\sum_{n=0}^{\infty} C_{n}, \\
\alpha \alpha_{\sigma} & =\sum_{n=0}^{\infty} C_{n} .
\end{aligned}
$$

For some Adomian polynomials, $A_{n}(\theta)$ are given by

$$
A_{n}\left(\theta_{0}, \theta_{1}, \theta_{2}, \ldots, \theta_{n}\right)=\frac{1}{n !} \frac{d^{n}}{d \lambda^{n}}\left[N\left(\sum_{n=0}^{\infty} \lambda^{n} \theta_{n}\right)\right]_{\lambda=0}, \quad n=0,1,2, \ldots
$$

Substituting equation (6) into both sides of equation (5) leads to

$$
\begin{aligned}
\sum_{n=0}^{\infty} \theta_{n}(\rho, \sigma, t)= & w(\rho, \sigma)-S^{-1}\left[u S\left[\left[\sum_{n=0}^{\infty} A_{n}\right]+\left[\sum_{n=0}^{\infty} B_{n}\right]\right]\right] \\
& +S^{-1}\left[u S\left[\frac{1}{R}\left(\nabla^{2}\left(\sum_{n=0}^{\infty} \theta_{n}(\rho, \sigma, t)\right)\right)\right]\right] \\
\sum_{n=0}^{\infty} \alpha_{n}(\rho, \sigma, t)= & h(\rho, \sigma)-S^{-1}\left[u S\left[\left[\sum_{n=0}^{\infty} C_{n}\right]+\left[\sum_{n=0}^{\infty} D_{n}\right]\right]\right] \\
& +S^{-1}\left[u S\left[\frac{1}{R}\left(\nabla^{2}\left(\sum_{n=0}^{\infty} \alpha_{n}(\rho, \sigma, t)\right)\right)\right]\right]
\end{aligned}
$$

To construct the recursive relation needed for the determination of the components $\left(\theta_{0}, \theta_{1}, \theta_{2}, \ldots, \theta_{n}\right)$ and $\left(\alpha_{0}, \alpha_{1}, \alpha_{2}, \ldots, \alpha_{n}\right)$, it is important to note that the Adomian method suggests that the zeroth components $\theta_{0}$ and $\alpha_{0}$ are usually defined by the functions $w(\rho, \sigma)$ and $h(\rho, \sigma)$.

Accordingly, the formal recursive relation is defined in (Figures 1 and 2).

$$
\begin{aligned}
\theta_{0}(\rho, \sigma, t)= & w(\rho, \sigma), \\
\theta_{k+1}(\rho, \sigma, t)= & -S^{-1}\left[u S\left[A_{k}+B_{k}\right]\right] \\
& +S^{-1}\left[u S\left[\frac{1}{R}\left(\nabla^{2}\left(\theta_{k}\right)\right)\right]\right], \quad k \geq 0, \\
\alpha_{0}(\rho, \sigma, t)= & h(\rho, \sigma), \\
\alpha_{k+1}(x, y, t)= & -S^{-1}\left[u S\left[C_{k}+D_{k}\right]\right] \\
& +S^{-1}\left[u S\left[\frac{1}{R}\left(\nabla^{2}\left(\alpha_{k}\right)\right)\right]\right], \quad k \geq 0 .
\end{aligned}
$$

Having determined these components, substitute it into $\theta(\rho, \sigma, t)=\sum_{n=0}^{\infty} \theta_{n}(\rho, \sigma, t)$ and $\alpha(\rho, \sigma, t)=\sum_{n=0}^{\infty} \alpha_{n}(\rho, \sigma, t)$ to obtain the solution in a series form.

\section{Application}

In this part, two examples are provided to illustrate the method.

Example 1. Consider the system of two-dimensional Burger's equation (1), with the following initial conditions [9]: 


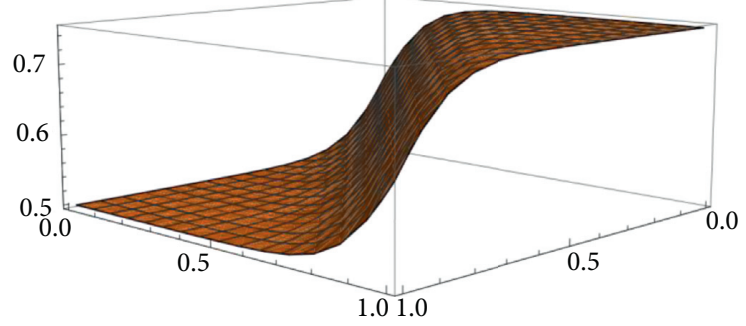

(a)

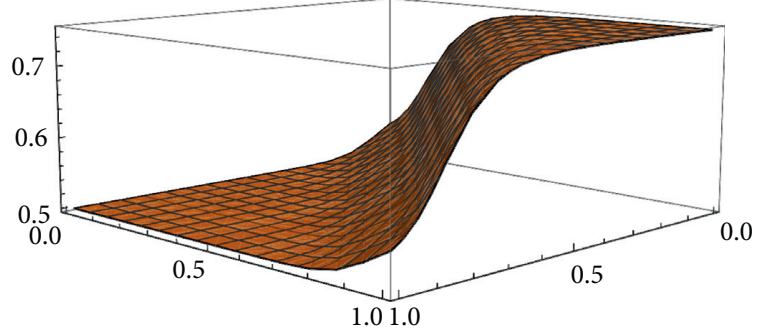

(b)

Figure 1: Distributions of approximation solutions for $\theta(\rho, \sigma, t)$ at (a) $t=0.01$ and (b) $t=0.5$ with $R=100$, for Example 2 .

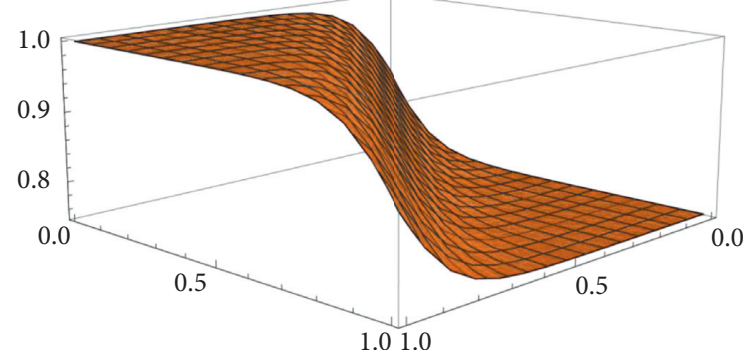

(a)

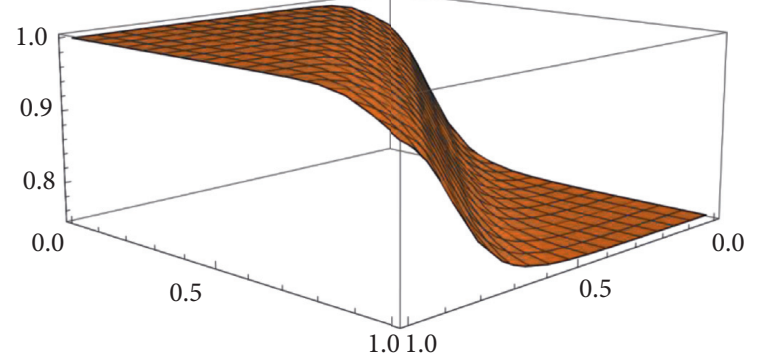

(b)

Figure 2: Distributions of approximation solutions for $\alpha(\rho, \sigma, t)$ at (a) $t=0.01$ and (b) $t=0.5$ with $R=100$, for Example 2 .

$$
\begin{aligned}
& \theta(\rho, \sigma, 0)=\rho+\sigma, \rho, \sigma \in E, \\
& \alpha(\rho, \sigma, 0)=\rho-\sigma, \rho, \sigma \in E .
\end{aligned}
$$

Solution. Subsequent to the discussion presented above, the system of equation (8) becomes

$$
\begin{aligned}
\sum_{n=0}^{\infty} \theta_{n}(\rho, \sigma, t)= & \rho+\sigma-S^{-1}\left[u S\left[\left[\sum_{n=0}^{\infty} A_{n}\right]+\left[\sum_{n=0}^{\infty} B_{n}\right]\right]\right] \\
& +S^{-1}\left[u S\left[\frac{1}{R}\left(\nabla^{2}\left(\sum_{n=0}^{\infty} \theta_{n}(\rho, \sigma, t)\right)\right]\right]\right. \\
\sum_{n=0}^{\infty} \alpha_{n}(\rho, \sigma, t)= & \rho-\sigma-S^{-1}\left[u S\left[\left[\sum_{n=0}^{\infty} C_{n}\right]+\left[\sum_{n=0}^{\infty} D_{n}\right]\right]\right] \\
& +S^{-1}\left[u S\left[\frac{1}{R}\left(\nabla^{2}\left(\sum_{n=0}^{\infty} \alpha_{n}(\rho, \sigma, t)\right)\right)\right] .\right.
\end{aligned}
$$

The recursive relation can be constructed from equation (11) given by

$$
\begin{aligned}
\theta_{0}(\rho, \sigma, t)= & \rho+\sigma, \\
\theta_{k+1}(\rho, \sigma, t)= & -S^{-1}\left[u S\left[A_{k}+B_{k}\right]\right] \\
& +S^{-1}\left[u S\left[\frac{1}{R}\left(\nabla^{2}\left(\theta_{k}\right)\right)\right]\right], \quad k \geq 0, \\
\alpha_{0}(\rho, \sigma, t)= & \rho-\sigma, \\
\alpha_{k+1}(x, y, t)= & -S^{-1}\left[u S\left[C_{k}+D_{k}\right]\right] \\
& +S^{-1}\left[u S\left[\frac{1}{R}\left(\nabla^{2}\left(\alpha_{k}\right)\right)\right]\right], \quad k \geq 0 .
\end{aligned}
$$

We get the next couple of components, and upon setting $R=1$, we have

$$
\begin{aligned}
& \left(\theta_{0}, \alpha_{0}\right)=(\rho+\sigma, \rho-\sigma), \\
& \left(\theta_{1}, \alpha_{1}\right)=(-2 \rho t,-2 \sigma t), \\
& \left(\theta_{2}, \alpha_{2}\right)=\left(2 \rho t^{2}+2 \sigma t^{2}, 2 \rho t^{2}-2 \sigma t^{2}\right), \\
& \left(\theta_{3}, \alpha_{3}\right)=\left(-4 \rho t^{3},-4 \sigma t^{3}\right), \\
& \left(\theta_{4}, \alpha_{4}\right)=\left(4 \rho t^{4}+4 \sigma t^{4}, 4 \rho t^{4}-4 \sigma t^{4}\right),
\end{aligned}
$$


TABLE 1: The (SDM) results for $\theta(\rho, \sigma, t)$ for first four approximations for $R=1$, with mesh points $\rho=0.1$ and $\sigma=0.1$, for Example 2 .

\begin{tabular}{lccr}
\hline$t$ & Exact $\theta(\rho, \sigma, t)$ & SDM $\theta(\rho, \sigma, t)$ & $\left|\theta_{\text {Exact }}-\theta_{\text {SDM }}\right|$ \\
\hline 0.05 & 0.6249023437698682 & 0.6249023437698682 & 0 \\
0.1 & 0.6248046876589456 & 0.6248046876589457 & $1.1102230 E-16$ \\
0.15 & 0.6247070317864406 & 0.6247070317864418 & $1.2212453 E-15$ \\
0.2 & 0.6246093762715608 & 0.6246093762715658 & $4.9960036 E-15$ \\
0.25 & 0.6245117212335117 & 0.6245117212335268 & $1.5099033 E-14$ \\
0.3 & 0.6244140667914967 & 0.6244140667915343 & $3.7636561 E-14$ \\
0.35 & 0.6243164130647162 & 0.6243164130647978 & $8.1601392 E-14$ \\
0.4 & 0.6242187601723671 & 0.6242187601725259 & $1.5876189 E-13$ \\
0.45 & 0.6241211082336422 & 0.6241211082339287 & $2.8654856 E-13$ \\
0.5 & 0.6240234573677299 & 0.624023457368215 & $4.8505644 E-13$ \\
\hline
\end{tabular}

TABLE 2: The (SDM) results for $\alpha(\rho, \sigma, t)$ for first four approximations for $R=1$, with mesh points $\rho=0.1$ and $\sigma=0.1$, for Example 2.

\begin{tabular}{lccr}
\hline$t$ & Exact $\alpha(\rho, \sigma, t)$ & $\operatorname{SDM} \alpha(\rho, \sigma, t)$ & $\left|\alpha_{\text {Exact }}-\alpha_{\text {SDM }}\right|$ \\
\hline 0.05 & 0.875097656230132 & 0.875097656230132 & 0 \\
0.1 & 0.875195312341054 & 0.875195312341054 & $1.1102230 E-16$ \\
0.15 & 0.875292968213559 & 0.875292968213558 & $1.2212453 E-15$ \\
0.2 & 0.875390623728439 & 0.875390623728434 & $4.9960036 E-15$ \\
0.25 & 0.875488278766488 & 0.875488278766473 & $1.5099033 E-14$ \\
0.3 & 0.875585933208503 & 0.875585933208466 & $3.7636561 E-14$ \\
0.35 & 0.875683586935284 & 0.875683586935202 & $8.1601392 E-14$ \\
0.4 & 0.875781239827633 & 0.875781239827474 & $1.5887291 E-13$ \\
0.45 & 0.875878891766358 & 0.875878891766071 & $2.8654856 E-13$ \\
0.5 & 0.87597654263227 & 0.875976542631785 & $4.8505644 E-13$ \\
\hline
\end{tabular}

TABLe 3: The (SDM) results for $\theta(\rho, \sigma, t)$, for first four approximations, and for $R=1$, with mesh points $\rho=0.3$ and $\sigma=0.1$, for Example 2 .

\begin{tabular}{lccr}
\hline$t$ & Exact $\theta(\rho, \sigma, t)$ & SDM $\theta(\rho, \sigma, t)$ & $\left|\theta_{\text {Exact }}-\theta_{\text {SDM }}\right|$ \\
\hline 0.05 & 0.6233399413556532 & 0.6233399413556536 & $4.4408921 E-16$ \\
0.1 & 0.6232423033622646 & 0.6232423033622709 & $6.3282712 E-15$ \\
0.15 & 0.6231446675140766 & 0.6231446675141092 & $3.2529535 E-14$ \\
0.2 & 0.6230470339301989 & 0.6230470339303031 & $1.0424994 E-13$ \\
0.25 & 0.6229494027297301 & 0.6229494027299877 & $2.5757174 E-13$ \\
0.3 & 0.6228517740317571 & 0.6228517740322975 & $5.4045657 E-13$ \\
0.35 & 0.6227541479553547 & 0.6227541479563675 & $1.0127454 E-12$ \\
0.4 & 0.6226565246195848 & 0.6226565144551626 & $1.7474910 E-12$ \\
0.45 & 0.622558904143496 & 0.6225588896714487 & $2.8308467 E-12$ \\
0.5 & 0.6224612866461229 & 0.622461286650486 & $4.3630655 E-12$ \\
\hline
\end{tabular}

and so on. Consequently, the solution in a series form is given by

$$
(\theta, \alpha)=\left(\begin{array}{c}
\rho\left(1+2 t^{2}+4 t^{4}+\cdots\right)-2 \rho t\left(1+2 t^{2}+\cdots\right)+\sigma\left(1+2 t^{2}+4 t^{4}+\cdots\right) \\
\rho\left(1+2 t^{2}+4 t^{4}+\cdots\right)-2 \sigma t\left(1+2 t^{2}+\cdots\right)-\sigma\left(1+2 t^{2}+4 t^{4}+\cdots\right)
\end{array}\right)
$$

and in a closed form it is

$$
(\theta(\rho, \sigma, t), \alpha(\rho, \sigma, t))=\left(\frac{\rho+\sigma-2 \rho t}{1-2 t^{2}}, \frac{\rho-\sigma-2 \sigma t}{1-2 t^{2}}\right)
$$

which is the exact solution of two-dimensional Burger's equations [9].

Example 2. Consider another system of Burger's equations (1), with the following initial conditions [9]: 
TABLE 4: The (SDM) results for $\alpha(\rho, \sigma, t)$, for first four approximations, and for $R=1$, with mesh points $\rho=0.3$ and $\sigma=0.1$, for Example 2 .

\begin{tabular}{lccr}
\hline$t$ & Exact $\alpha(\rho, \sigma, t)$ & $\operatorname{SDM} \alpha(\rho, \sigma, t)$ & $\left|\alpha_{\text {Exact }}-\alpha_{\text {SDM }}\right|$ \\
\hline 0.05 & 0.876660058644347 & 0.876660058644346 & $4.4408921 E-16$ \\
0.1 & 0.876757696637735 & 0.876757696637729 & $6.3282712 E-15$ \\
0.15 & 0.876855332485923 & 0.876855332485891 & $3.2529535 E-14$ \\
0.2 & 0.876952966069801 & 0.876952966069697 & $1.0424994 E-13$ \\
0.25 & 0.87705059727027 & 0.877050597270012 & $2.5757174 E-13$ \\
0.3 & 0.877148225968243 & 0.877148225967703 & $5.4045657 E-13$ \\
0.35 & 0.877245852044645 & 0.877245852043633 & $1.0127454 E-12$ \\
0.4 & 0.877343475380415 & 0.877343485544838 & $1.7474910 E-12$ \\
0.45 & 0.877441095856504 & 0.877441095853673 & $2.8309577 E-12$ \\
0.5 & 0.877538713353877 & 0.877538713349514 & $4.3630655 E-12$ \\
\hline
\end{tabular}

$$
\begin{aligned}
& \theta(\rho, \sigma, 0)=\frac{3}{4}-\frac{1}{4\left(1+e^{(R(\sigma-\rho) / 8)}\right)} \\
& \alpha(\rho, \sigma, 0)=\frac{3}{4}+\frac{1}{4\left(1+e^{(R(\sigma-\rho) / 8)}\right)}
\end{aligned}
$$

with the exact solutions:

$$
\begin{aligned}
& \theta_{0}(\rho, \sigma, t)=\frac{3}{4}-\frac{1}{4\left(1+e^{(R(-\rho+\sigma) / 8)}\right)}, \\
& \alpha_{0}(\rho, \sigma, t)=\frac{3}{4}+\frac{1}{4\left(1+e^{(R(-\rho+\sigma) / 8)}\right)}, \\
& \theta_{1}(\rho, \sigma, t)=-\frac{e^{(1 / 8) R(-\rho+\sigma)} R t}{64\left(1+e^{(1 / 8) R(-\rho+\sigma)}\right)^{3}}-\frac{e^{(1 / 4) R(-\rho+\sigma)} R t}{64\left(1+e^{(1 / 8) R(-\rho+\sigma)}\right)^{3}}+\frac{e^{(1 / 8) R(-\rho+\sigma)} R t}{128\left(1+e^{(1 / 8) R(-\rho+\sigma)}\right)^{2}}, \\
& \alpha_{1}(\rho, \sigma, t)=\frac{e^{(1 / 8) R(-\rho+\sigma)} R t}{64\left(1+e^{(1 / 8) R(-\rho+\sigma)}\right)^{3}}+\frac{e^{(1 / 4) R(-\rho+\sigma)} R t}{64\left(1+e^{(1 / 8) R(-\rho+\sigma)}\right)^{3}}-\frac{e^{(1 / 8) R(-\rho+\sigma)} R t}{128\left(1+e^{(1 / 8) R(-\rho+\sigma)}\right)^{2}}, \\
& \theta_{2}(\rho, \sigma, t)=-\frac{e^{(R \rho / 8)+(R \sigma / 8)}\left(-e^{(R \rho / 8)}+e^{(R \sigma / 8)}\right) R^{2} t^{2}}{8192\left(e^{(R \rho / 8)}+e^{(R \sigma / 8)}\right)^{3}}, \\
& \alpha_{2}(\rho, \sigma, t)=\frac{e^{(R \rho / 8)+(R \sigma / 8)}\left(-e^{(R \rho / 8)}+e^{(R \sigma / 8)}\right) R^{2} t^{2}}{8192\left(e^{(R \rho / 8)}+e^{(R \sigma / 8)}\right)^{3}}, \\
& \alpha_{3}(\rho, \sigma, t)=\frac{e^{(R \rho / 8)+(R \sigma / 8)}\left(e^{(R \rho / 4)}+e^{(R \rho / 4)}-4 e^{(R \rho / 8)+(R \sigma / 8)}\right) R^{3} t^{3}}{786432\left(e^{(R \rho / 8)+(R \sigma / 8)}\right)^{4}} . \\
& -\frac{e^{(R \rho / 8)+(R \sigma / 8)}\left(e^{(R \rho / 4)}+e^{(R \sigma / 4)}-4 e^{(R \rho / 8)+(R \sigma / 8)}\right) R^{3} t^{3}}{786432\left(e^{(R \rho / 8)+(R \sigma / 8)}\right)^{4}},
\end{aligned}
$$

$$
\begin{aligned}
& \theta(\rho, \sigma, t)=\frac{3}{4}-\frac{1}{4\left(1+e^{(R(4 \sigma-4 \rho-t) / 32)}\right)}, \\
& \alpha(\rho, \sigma, t)=\frac{3}{4}+\frac{1}{4\left(1+e^{(R(4 \sigma-4 \rho-t) / 32)}\right)} .
\end{aligned}
$$

Solution. Using the previous aforesaid discussion, we get
Therefore, the solution $\theta(\rho, \sigma, t)$ and $\alpha(\rho, \sigma, t)$ in the series form is given by

$$
\begin{gathered}
\theta(\rho, \sigma, t)=\theta_{0}(\rho, \sigma, t)+\theta_{1}(\rho, \sigma, t)+\theta_{2}(\rho, \sigma, t)+\theta_{3}(\rho, \sigma, t), \\
\alpha(\rho, \sigma, t)=\alpha_{0}(\rho, \sigma, t)+\alpha_{1}(\rho, \sigma, t)+\alpha_{2}(\rho, \sigma, t)+\alpha_{3}(\rho, \sigma, t) .
\end{gathered}
$$




\section{Conclusion}

In this paper, SDM had been successfully applied to find the solutions of the system of two-dimensional nonlinear Burger's equations. The numerical studies showed that SDM offers accurate results for two-dimensional nonlinear Burger's equations in comparison with another analytical methods. This fact is shown in the second example. Therefore, this method may be a favourable method to solve other nonlinear partial differential equations.

\section{Data Availability}

No data were used to support this study.

\section{Conflicts of Interest}

The authors declare that they have no conflicts of interest.

\section{References}

[1] J. M. Burger, "A mathematical model illustrating the theory of turbulence," Advances in Applied Mechanics, vol. 1, pp. 171199,1948

[2] J. D. Cole, "On a quasi-linear parabolic equation occurring in aerodynamics," Quarterly of Applied Mathematics, vol. 9, no. 3, pp. 225-236, 1951.

[3] E. Hopf, "The partial differential equation $u_{t}+u u_{x}=\mu u_{x x}$.", Communications on Pure and Applied Mathematics, vol. 3, no. 3, pp. 201-230, 1950.

[4] P. D. Lax, Hyperbolic Systems of Conservation Laws and the Mathematical Theory of Shock Waves, SIAM, Philadelphia, PA, USA, 1973.

[5] J. Smoller, Shock Waves and Reaction-Diffusion Equations, Springer-Verlag, Berlin, Germany, 1983.

[6] R. Abazari and A. Borhanifar, "Numerical study of the solution of the Burgers and coupled Burgers equations by a differential transformation method," Computers \& Mathematics with Applications, vol. 59, no. 8, pp. 2711-2722, 2010.

[7] A. Molabahrami, F. Khani, and S. Hamedi-Nezhad, "Soliton solutions of the two-dimensional KdV-Burgers equation by homotopy perturbation method," Physics Letters A, vol. 370, no. 5-6, pp. 433-436, 2007.

[8] M. Inc, "On numerical solution of Burger's equation by homotopy analysis method," Physics Letters A, vol. 372, no. 4, pp. 356-360, 2008.

[9] J. Biazar and H. Aminikhah, "Exact and numerical solutions for non-linear Burger's equation by VIM," Mathematical and Computer Modelling, vol. 49, pp. 1394-1400, 2009.

[10] M. Dehghan, A. Hamidi, and M. Shakourifar, "The solution of coupled Burger's equations using Adomian-pade technique," Applied Mathematics and Computation, vol. 189, pp. 10341047, 2007.

[11] A. Gorguis, "A comparison between Cole-Hopf transformation and the decomposition method for solving Burger's equations," Applied Mathematics and Computation, vol. 173, no. 1, pp. 126-136, 2006.

[12] A. Alharbi and E. S. Fahmy, "Adm-pade solutions for generalized Burger's and Burger's-Huxley systems with two coupled equations," Journal of Computational and Applied Mathematics, vol. 233, no. 8, pp. 2071-2080, 2010.

[13] B. K. Singh and P. Kumar, "A novel approach for numerical computation of Burger's equation in $(1+1)$ and $(2+1)$ dimensions," Alexandria Engineering Journal, vol. 55, no. 4, pp. 3331-3344, 2016.

[14] A. H. A. Ali and G. A. Gardner, "A collocation solution for Burger's equation using cubic B-spline finite elements," Computer Methods in Applied Mechanics and Engineering, vol. 100, no. 3, pp. 325-337, 1992.

[15] M. Basto, V. Semiao, and F. Calheiros, "Dynamics and synchronization of numerical solutions of the Burger's equation," Journal of Computational and Applied Mathematics, vol. 231, no. 2, pp. 793-806, 2009.

[16] G. Zhao, X. Yu, and R. Zhang, "The new numerical method for solving the system of two-dimensional Burger's equations," Computers \& Mathematics with Applications, vol. 62, no. 8, pp. 3279-3291, 2011.

[17] M. A. Gondal, S. I. Batool, and M. Khan, "A novel fractional Laplace decomposition method for chaotic systems and the generation of chaotic sequences," Journal of Vibration and Control, vol. 20, no. 16, pp. 2530-2535, 2014.

[18] M. Khan, F. Soleymani, and M. A. Gondal, "A new analytical solution procedure for the motion of a spherical particle in a plane Couette flow," Zeitschrift für Naturforschung A, vol. 68, no. 5, pp. 319-326, 2013.

[19] A. Salah, M. Khan, and M. A. Gondal, "A novel solution procedure for fuzzy fractional heat equations by homotopy analysis transform method," Neural Comput \& Applic, vol. 23, no. 2, pp. 269-271, 2013.

[20] M. Khan, M. A. Gondal, and K. Omrani, "A new analytical approach to two-dimensional magneto-hydrodynamics flow over a nonlinear porous stretching sheet by Laplace Padé decomposition method," International Journal of Results in Mathematics, vol. 63, pp. 289-301, 2013.

[21] M. A. Gondal, A. Salah, M. Khan, and S. I. Batool, "A novel analytical solution of a fractional diffusion problem by homotopy analysis transform method," Neural Comput \& Applic, vol. 23, no. 6, pp. 1643-1647, 2013.

[22] M. Khan, M. A. Gondal, and S. I. Batool, "A new modified Laplace decomposition method for higher order boundary value problems," Computational and Mathematical Organization Theory, vol. 19, no. 4, pp. 446-459, 2013.

[23] M. Khan and M. A. Gondal, "A reliable treatment of Abel's second kind singular integral equations," Applied Mathematics Letters, vol. 25, no. 11, pp. 1666-1670, 2012.

[24] M. Khan, M. A. Gondal, and S. Kumar, "A new analytical solution procedure for nonlinear integral equations," Mathematical and Computer Modelling, vol. 55, no. 7-8, pp. 1892-1897, 2012.

[25] M. Khan and M. A. Gondal, "New computational dynamics for magnetohydrodynamics flow over a nonlinear stretching sheet," Zeitschrift für Naturforschung A, vol. 67, no. 5, pp. 262-266, 2012.

[26] M. Khan, M. A. Gondal, and S. I. Batool, "A novel analytical implementation of nonlinear volterra integral equations," Zeitschrift Fur Naturforschung, vol. 67, no. 12, pp. 674-678, 2012.

[27] M. Khan, M. A. Gondal, I. Hussain, and S. Karimi Vanani, "A new comparative study between homotopy analysis transform method and homotopy perturbation transform method on a semi infinite domain," Mathematical and Computer Modelling, vol. 55, no. 3-4, pp. 1143-1150, 2012.

[28] M. Khan and M. Hussain, "Application of Laplace decomposition method on semi-infinite domain," Numerical Algorithms, vol. 56, no. 2, pp. 211-218, 2011.

[29] M. Khan and M. A. Gondal, "Homotopy perturbation padé transform method for blasius flow equation using He's 
polynomials," International Journal of Nonlinear Sciences and Numerical Simulation, vol. 12, no. 1-7, 2011.

[30] M. A. Gondal and M. Khan, "Homotopy perturbation method for nonlinear exponential boundary layer equation using Laplace transformation, He's polynomials and Pade technology," International Journal of Nonlinear Science and Numerical Simulation, vol. 11, no. 12, pp. 1145-1153, 2010.

[31] S. A. Ahmed, "A comparison between modified Sumudu decomposition method and homotopy perturbation method," Applied Mathematics, vol. 9, no. 3, pp. 199-206, 2018.

[32] S. Ahmed and T. Elzaki, "The solution of nonlinear Volterra integro-differential equations of second kind by combine Sumudu transforms and Adomian decomposition method," International Journal of Advanced and Innovative Research, vol. 2, no. 12, pp. 90-93, 2013.

[33] S. Ahmed and T. Elzaki, "A comparative study of Sumudu decomposition method and Sumudu projected differential transform method," World Applied Sciences Journal, vol. 31, no. 10, pp. 1704-1709, 2014.

[34] S. Ahmed and T. Elzaki, "Solution of heat and wave-like equations by adomian decomposition Sumudu transform method," British Journal of Mathematics \& Computer Science, vol. 8, no. 2, pp. 101-111, 2015.

[35] S. Ahmed and T. Elzaki, "On the comparative study integro-differential equations using difference numerical methods," Journal of King Saud University-Science, vol. 32, no. 1, pp. 84-89, 2018.

[36] D. Kumar, J. Singh, and S. Rathore, "Sumudu decomposition method for nonlinear equations," International Mathematical Forum, vol. 7, no. 11, pp. 515-521, 2012. 